\title{
Design of the quarantine surveillance for non-indigenous species of invertebrates on Barrow Island
}

\author{
Peter Whittle ${ }^{1,2}$, Frith Jarrad ${ }^{1,2}$ and Kerrie Mengersen ${ }^{1,2}$ \\ ' Queensland University of Technology, Science and Engineering Faculty, GPO Box 2434, Brisbane \\ Queensland 4001, Australia. Email: peter.whittle@qut.edu.au \\ 2 Plant Biosecurity Cooperative Research Centre, LPO Box 5012, Bruce ACT 2617, Australia.
}

\begin{abstract}
The Ministerial conditions for regulatory approval for the Gorgon gas project on Barrow Island included a quarantine surveillance program having detection power of 0.8 for non-indigenous species of terrestrial invertebrates, vertebrates and plants. No method was available for design of such a program, so we developed a new method and designed surveillance systems that were implemented successfully in 2010-11 for the first of four years over the construction period. Here we describe the method and outline the invertebrate surveillance system, after the experience of the first year. We discuss a set of issues that characterised the design problem, which we consider typical of many surveillance applications. We suggest that the method is broadly applicable for objective design of surveillance, for biosecurity and other settings.
\end{abstract}

KEYWORDS: surveillance, biosecurity, monitoring, non-indigenous species

\section{INTRODUCTION}

The quarantine surveillance program for terrestrial non-indigenous species (NIS) of invertebrates in the Gorgon gas project on Barrow Island (BWI) is part of a larger program covering vertebrates and plants as well. This surveillance program is one of the requirements of the Gorgon environmental approval (Government of Western Australia 2007), which specified a statistical power of detection for NIS of at least 0.8. The use of statistical power for biosecurity surveillance system (SS) design had not been reported before. Moreover, even though this concept is used frequently in other branches of science, it is typically employed for more traditional experimental setups, far removed from the BWI context. As a result, creating a surveillance design that satisfied statistical power for BWI required development of a new methodology. The approach has been described with application to a single species by Barrett et al. (2010) (big-headed ant, Pheidole megacephala) and Jarrad et al. (2011) (black rat, Rattus rattus). Whittle et al. (2009) reported the power SS design method for multiple species with application to invertebrates, vertebrates and plants, and this has been further reported for vertebrates with discussion of ecological aspects (Jarrad et al. 2010) and invertebrates with a focus on risk aspects
(Whittle et al. 2013). Here we discuss the design issues and present the integrated invertebrate SS, to supplement the contents of this volume.

Biosecurity surveillance is conducted to provide assurance that a threat is not present, or to detect an incursion early enough that an effective response can be mounted, enabling eradication and preventing unacceptable damage (Mehta et al. 2007; Cacho et al. 2010). As a corporate or regulatory activity with legal implications, this requires rational design based on objectives (Mengersen and Whittle 2011). However, surveillance is frequently designed with budget as the first consideration and often with no statistical input at all (Marsh and Trenham 2008). We consider that the notion of surveillance design is often confused with development and effective performance of detection methods. By using statistical design (and analysis), detection methods can be deployed optimally to provide the surveillance data required to determine the degree to which corporate or regulatory responsibilities have been met, or what resources are required for surveillance to meet them.

A common statistical approach to surveillance design is to use a confidence specification (e.g. the sample size required to give $95 \%$ confidence of detecting an NIS if it is present at $1 \%$ prevalence) (Cannon and Roe 1982). An alternative approach 


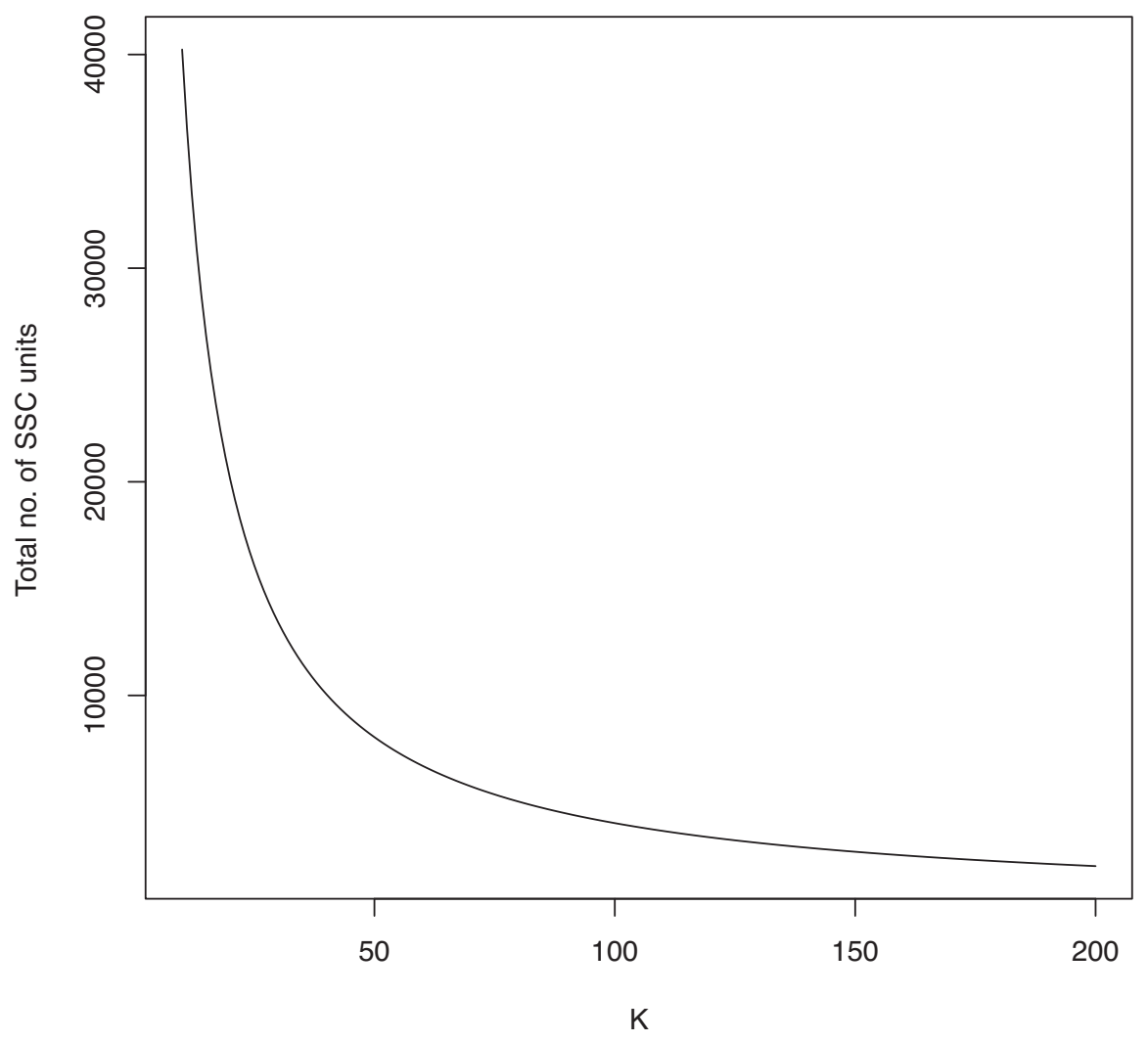

FIGURE 1 The effect of changing the tolerable population size ( $K$ ) on the number of required surveillance system component (SSC) units for power of 0.8 .

is to design to a specified statistical power (here, the probability of the SS detecting an NIS, given it is present at a threshold population size $K^{*}$ (equivalent to Cannon's (2002) design prevalence).

Power is an intuitive design standard for surveillance. We can consider the baseline hypothesis to be that the pest is absent. The key concern of inadequate surveillance is the Type II error, the probability of falsely accepting this hypothesis, by declaring the NIS to be absent when it is, in fact, present. Power can be increased, for example, by improving the detection methodology and/or increasing the number of surveillance units. A Type I error (declaring the NIS to be present when in fact it is absent, i.e. a false positive) would be a diagnostic error and is not usually a matter of the surveillance design per se (Martin et al. 2007).

For the BWI program, satisfaction of a high statistical power therefore required calculation of the probability of the SS detecting an NIS, given it is present at a threshold population size $K$. Figure 1 shows that, for a detection power of 0.8 , there is a sharp increase in the number of surveillance units required for $K$ between 10 and about 200, the optimal range. A similar calculation can be made to demonstrate that improving the detection methodology (i.e. increasing the probability that each individual surveillance unit detects the pest if it is present) also increases the power. Thus, the setting of power is a pure risk decision - how much to spend on surveillance set against the probability of detection.

BWI shares a feature with many quarantine surveillance contexts in having an area that is too large to survey adequately, but an opportunity arises from risk rarely being homogeneous across a surveillance frame. For a given power and surveillance area, the proportional relationship between $K$ and the amount of surveillance required has two further key implications - large returns can be gained from focusing surveillance in high risk areas, and low-intensity surveillance in the remaining areas can be of negligible value (Whittle et al. 2013). For these reasons, the BWI design problem was simplified by the rigorous quarantine restrictions on entry opportunities (Chevron Australia Pty Ltd 2011), making it extremely unlikely that entry would occur on the majority of the island. The task was also simplified by the extensive geographical information system (GIS) on BWI. A further consideration was that an escape could be detected in the additional ongoing

\footnotetext{
* $\mathrm{K}$ is defined as a number of individuals or independent groups which is large enough to be detectable, but not too large
} to be eradicable or to pose significant environmental consequences. 
environmental monitoring program that will be conducted across the island in areas that are not at high risk.

In quarantine, because the number of threats is often very large (e.g. over 120,000 alien plant, animal and microbe species have been recorded in six countries (Pimentel et al. 2001)), surveillance is often risk-targeted on species considered more likely to invade or cause substantial damage. Many approaches have been employed to try to identify these (Kolar and Lodge 2001; Whittle et al. 2013), but the BWI problem lacked defined targets - all NIS were unacceptable and had to be detected. Thus, we needed to devise an SS that could detect any and every invertebrate species. We considered that this could be done by designing the SS to detect a set of exemplar species, focusing not on their invasion risk, but on their detection methods. If we designed an SS for each exemplar using a variety of detection methods (termed SS components (SSCs) after Martin et al. (2007)), then integrated the exemplar SSs, we would have a universal, multi-component SS. An ideal exemplar species would have been studied and reported extensively in regard to its ecology and detection. Each should have several well known SSCs, so that the combined SSCs of the exemplar set would have no deficiencies for detecting all invertebrates.

Combining data from SSCs presented a variety of problems for rational design. The first was how to identify (and optimise) the contribution to total SS power made by each SSC, for example, the relative sensitivity of detection of pitfall traps and window traps for a given species. Martin et al. (2007) used scenario trees to analyse the sensitivity of detection of animal diseases using several data sources, but this method did not lend itself to design of a new system, nor was another method available (Shmueli and Fienberg 2006; Salman 2008). The method of Martin et al. (2007) also indicated an opportunity at BWI, to use the detection power of construction workers on the island; while the detection sensitivity (power) of one worker may be negligible, the surveillance value of many may be useful (Cacho et al. 2010). The next problem with combining SSCs concerned optimal cost. On BWI, we were fortunate to be relatively unencumbered by overall cost, because of the extremely high value of the Gorgon project, although the overall cost of surveillance should be balanced against expenditure on exclusion activities (Moore et al. 2010). However, use of particular SSCs was constrained in ways that could be defined broadly as cost, e.g. diagnostic expertise was limited in availability, and removing large numbers of specimens or substrate would cause unacceptable environmental damage (Jarrad et al. 2010; Whittle et al. 2013).

To design an SS, we need to understand the model (what we are looking at and how), the parameters (of the sampling frame, the SSCs, the target etc.) and the data (its variability), but each of these will be surrounded by uncertainty, affecting the robustness of the design (Mengersen and Whittle 2011). Rather than delaying progress through the precautionary principle while collecting information to address uncertainty (De Sadeleer 2007), expert opinion is often used to fill information gaps in ecology (Marsh and Trenham 2008). This can be done in a Bayesian framework through elicitation, using a GIS to identify a probability surface for occurrence of a species (Denham and Mengersen 2007; Low Choy et al. 2009; Stanaway et al. 2011). However, it is important to later update models made with extensive information gaps, by testing its underpinning assumptions (McCann et al. 2006).

In our SS design method, we have addressed all of the above issues of a statistical design requirement, a large surveillance area, multiple targets, combining multiple sources of data, cost optimisation and information gaps. Here we present the integrated, multiple species invertebrate SS, to provide context for the content of this book. At the time of writing, surveillance had been conducted for the first year of the four-year construction period, so only limited results are presented.

\section{MATERIALS AND METHODS}

The SS design method is based on the concept that a wide range of SSCs can be selected and combined to detect all potential NIS. To do this, we selected a set of widely differing species as exemplars of other species, designed an SS for each one, then integrated these exemplar SSs to a single invertebrate NIS SS.

For simplicity of implementation, the integrated SS plan consisted of a list of SSCs and the number of units of each that had to be deployed in specified locations (entry points and risk zones) during the surveillance period. This provided a clear specification for Chevron and its contractors to use in organising, implementing, reporting and reviewing BWI surveillance.

Decisions about choices of exemplar species and SSCs were made using standard elicitation methods (Low Choy et al. 2009) in five workshops over 18 months, by a group of biologists with expertise in invertebrate ecology and detection, in environmental and biosecurity contexts. Workshops were commenced with discussion of surveillance design issues and principles, and the context of BWI entomology, informed by the very extensive baseline surveillance there (Callan et al. 2011) (Majer et al. 2013). As the set of exemplars and the set of SSCs for each was identified and 
characterised, they were reviewed repeatedly for coverage of the full range of potential NIS. Detailed data sheets were prepared for each exemplar species. Estimates were elicited using structured questions and group discussion until consensus was reached. All workshops were carefully minuted and checked with participants, and estimates, other information and the emerging SS designs were returned to them for comment. Thus the exemplar SSs were designed in several iterations until the experts were satisfied with the final array of SSCs and the units of each.

A generalised NIS detection model (Figure 2) helped to clarify the design issues. Detection outcomes are determined by detectability (the probability that the SS will detect the target, given it is present) and occupancy (the target's presence, distribution and abundance in the surveillance frame). Each SSC unit (a single iteration conducted to standard protocol) has the characteristics of footprint (the area of the surveillance frame it detects in $\mathrm{m}^{2}$ ), sensitivity (defined above, sigma ( $\sigma)$ unitless) and cost (defined above, broad sense, unitless). The 'standard protocol' implies the SS will be deployed in sites (spatial element) and at times (temporal element) that will optimise detection (Callan et al. 2011). The probability of occupancy of a site is determined by entry and establishment factors, the conventional components of pest risk analysis (IPPC 2007). Entry factors include the type, source, volume and treatment of materials that might assist entry of an NIS. Establishment or habitat suitability factors include the availability of food, moisture, shelter and competition that determine whether an NIS, once entered, can form a self-perpetuating population. It can be seen that these are conditional probabilities, and thus they are multiplied together to calculate the probability of detection.
The expert group estimated $K$ for each exemplar species, which is required to generate the SS. To do this, they considered the feasibility and cost of detecting it, its expected rate of population growth on BWI, and the potential risk of detecting it late.

The suitability of SSCs for BWI from amongst the wide range available for invertebrates (Augustin et al. 2012; Quilici et al. 2012) has been informed by the extensive experience of Callan et al. (2011) (Majer et al 2013). The experts selected a set of SSCs for each exemplar species, ensuring that the integrated list would detect all invertebrate taxa. They characterised the SSCs for the technical parameters footprint, $\sigma$ and cost. Cost of SSCs were estimated initially from the time involved in collecting samples (high due to the remoteness of and limited access to BWI) and diagnostic costs (Bisevac and Majer 2002). These initial cost estimates needed to be weighted up or down, or capped, for certain SSCs for reasons such as limited availability of diagnostic expertise, or unacceptability of removing large quantities of fauna or substrate from the BWI environment. The SSC 'unskilled workers' needed to be downweighted, as its nil-incremental cost caused it to dominate the SS otherwise.

The SS needed to be performed over a specified period, for which a statement of NIS freedom, based on power of 0.8 , could be made. Although the full construction period of four years could be used, several factors should be taken into account such as epidemiology and the rate of population growth, the rate of surveillance itself, economic and political consequences of a detection, and management factors of the construction project (Martin et al. 2007). The expert panel decided on a one-year time period, because most NIS would not be expected to multiply rapidly on BWI, their detectability was

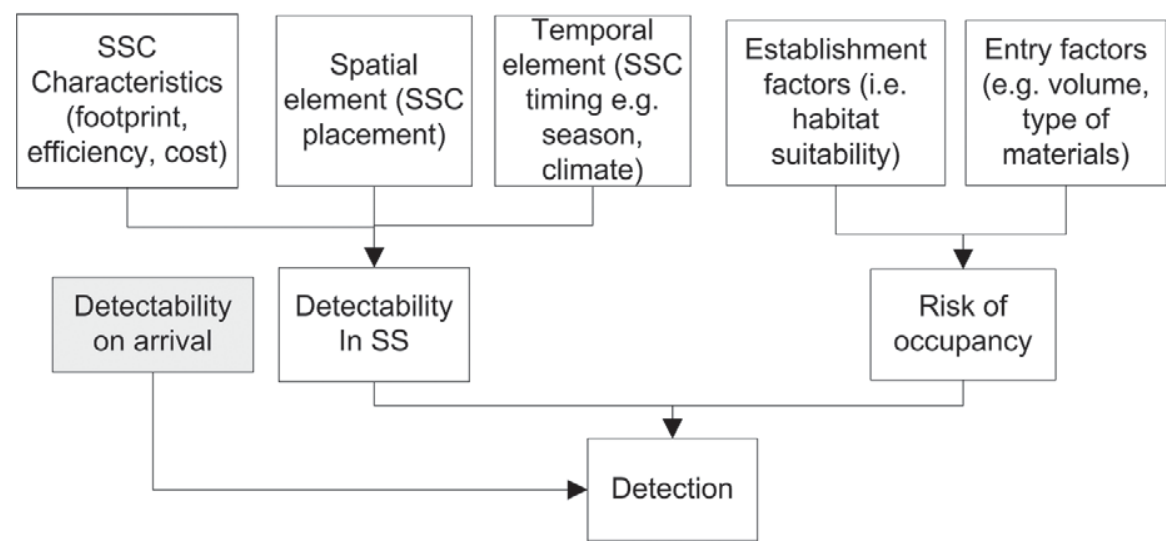

FIGURE 2 Generalised model of detection of non-indigenous species and the factors contributing to it. SSC = 'surveillance system component' (e.g. pitfall trap, biologist survey, etc.). Occupancy is equivalent to likelihood of entry and establishment and hence is a key component of risk. The scope of this study commences after arrival and hence detectability on arrival is outside the scope of this study, but is illustrated for clarity. 
likely to vary through and between years due to seasonal factors, and a one-year period would fit with normal management systems. Thus, the SS was designed to be deployed over the course of a year, taking into account seasonal factors to maximise detection probability.

To allocate surveillance effort spatially using risk, we built a decision tree (Figure 3), using Insight Tree $\odot$ software (Lehmann and Lehmann 2008), which calculates conditional probabilities of detection given presence $(\operatorname{Pr}[\mathrm{D}+\mid \mathrm{P}])$ from the probabilistic steps along each branch (by multiplication). Probabilities were estimated for each exemplar species by the experts in workshops. Assuming the island had limited entry points for NIS (see above) and was heterogeneous for NIS habitat suitability, we proposed three risk zones:

- Zone 1 (Z1) - an entry point, where NIS could potentially enter and establish;

- Zone 2 (Z2) - a buffer zone around Z1, with habitat suitable for NIS establishment; and

- Zone 3 (Z3) - all other areas, suitable or otherwise for establishment.

We postulated that an NIS was extremely unlikely to enter $\mathrm{Z} 1$ and become detectable only in $Z 3$, so that surveillance should only be allocated to $\mathrm{Z1}$ and Z2. Furthermore, this NIS would potentially be detected in the separately organised ongoing environmental monitoring program to be carried out across the island), or it may re-enter Zones 1 or 2 and become detectable by the SS. Thus the branch probabilities informed allocation of the SS between Z1 and Z2. The combined area of Zones 1 and 2 became the surveillance frame $(F)$ required for calculating the SS, and the individual zone areas were required for allocating the SS for deployment. The detection scenario commences with whether or not the NIS is detected on its arrival on the island, after which it may follow one of the following scenarios:

1. Does not leave Z1; or

2. Spreads to $\mathrm{Z} 2$ but also remains present in $\mathrm{Z1}$; or

3. Spreads to $Z 2$ and leaves $Z 1$.

Scenarios 1 and 2 can be detected by surveillance in Z1, hence they can be pooled. Thus the relative apportionment to $Z 1$ is given by $\operatorname{Pr} 1+\operatorname{Pr} 2$, and that for Z2 from Pr3.

Risk maps for each exemplar species were prepared using the Analytical Hierarchy Process (AHP) (Saaty 1987) by Alex Nietrzeba (Curtin University, personal communication), based on the concept of occupancy and its components (Figure 2), as described by Barrett et al. (2010) and Jarrad et al. (2010) and outlined here. The key attributes entry and establishment were characterised in terms of the factors (criteria) expected to contribute to
TABLE $1 \quad$ Scale of linguistic expressions used to make pair-wise comparisons in Analytical Hierarchy Process. Experts nominate their preference and this is translated to a numerical value for calculating relative importance weights.

\begin{tabular}{ll}
$\begin{array}{l}\text { Linguistic expression of relative } \\
\text { importance of one member } \\
\text { of comparison pair relative to } \\
\text { another }\end{array}$ & $\begin{array}{l}\text { Number assigned } \\
\text { to linguistic } \\
\text { expression }\end{array}$ \\
\hline $\begin{array}{l}\text { Equal preference or } \\
\text { indifference }\end{array}$ & 1 \\
Weak preference & 3 \\
Strong preference & 5 \\
$\begin{array}{l}\text { Demonstrated preference } \\
\text { Absolute preference }\end{array}$ & 7 \\
Intermediate Values & 9 \\
\hline
\end{tabular}

them. The experts in workshops made pairwise comparisons of 'preference' between each entry point and between the criteria of establishment, using a linguistic scale that was related to a numerical scale (Table 1). Relative importance weights were calculated for each criterion, enabling entry and establishment to be mapped with the BWI geographical information system (GIS). Then, these attributes were multiplied together to build an occupancy risk map, in which GIS polygons with high risk (both attributes high) were classed as Z1, those of moderate risk (positive but not high probability for either or both attributes) were Z2, and all other areas were Z3. Examples of risk maps (for vertebrates) can be viewed in Jarrad et al. (2010).

We developed a spreadsheet to calculate the SS for each exemplar, using the statistical model described here and encapsulated in Equation 1 (Barrett et al. 2010; Jarrad et al. 2010; Jarrad et al. 2011). It calculates a relative weight for each SSC from its footprint, sensitivity $(\sigma)$ and cost in combination with those of the other selected SSCs. The SS (number of units of each SSC) is calculated from the relative weights and $F$. Each exemplar species has a risk $\left(R_{j}\right)$ of occupancy in risk zone $j=1,2$. Each of the $i$ types of SSC $i$ in the SS has a different sensitivity $\left(\sigma_{i}\right)$ and cost $\left(C_{i}\right)$. Assuming that each SSC $_{i}$ is independent, it contributes a 'utility' $\left(\beta_{i}\right)$ to the overall power $(\beta)$. The number of SSCs of type $i$ that should be deployed in zone $j$ is given by the proportional areas of each zone. 


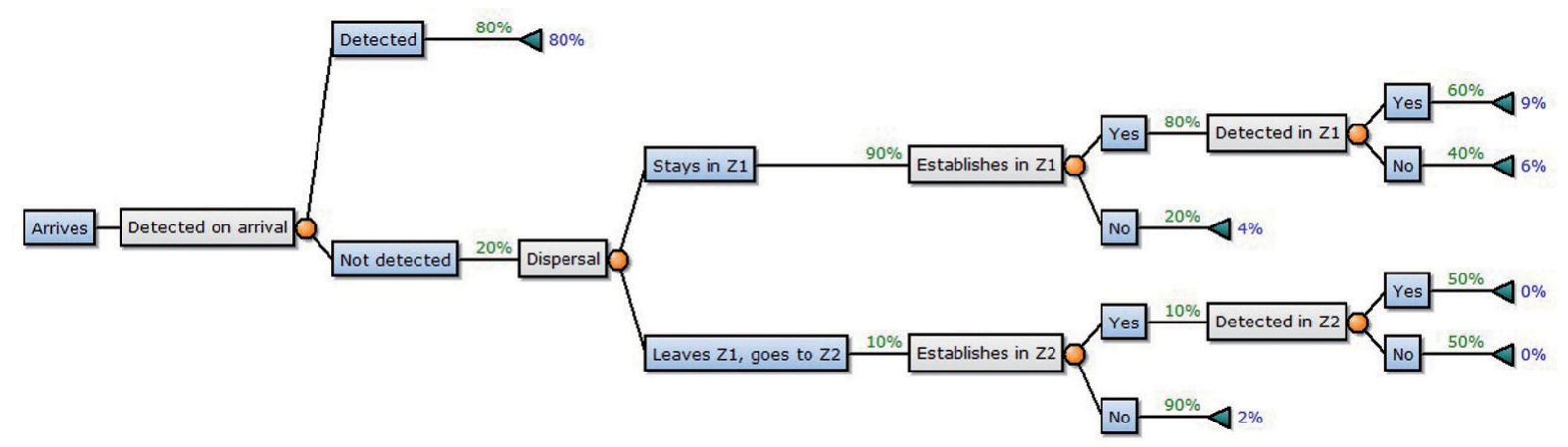

FIGURE 3 Decision tree of generic invertebrate dispersal pathways on Barrow Island. Zone 1 refers to the higher risk zones where the initial introduction occurs. Zone 2 refers to a lower risk area to which the NIS may escape. The tree was produced using the software InsightTree (Lehmann and Lehmann 2008).

\section{EQUATION 1}

Having developed each exemplar species SS, they are deployed in two stages, based on a proportional stratified design. The first stage is spatial deployment using the GIS, between the discrete entry points and risk zones in relation to their relative areas and the relative weighting allocations to $\mathrm{Z} 1$ and $\mathrm{Z} 2$. At this point, the exemplar SSs were integrated to a single SS, by overlaying and for each polygon for each SSC, selecting the largest unit allocation. If the number of units of an SSC was not a whole number, it was rounded up to the nearest unit.

The second stage of deployment is undertaken by surveillance staff, who select the precise location and timing for each SSC unit to optimise detectability. For example, 10 SSC units could be distributed to all required localities simultaneously and randomly, or if detectability was expected to be higher in particular places or at particular times, they were expected to select these to best practice. The expert panel had assumed this competence in estimating the model inputs and the assumptions underpinning design power. Selecting and documenting the SSC protocol was the responsibility of the implementing biologists, and was outside the scope of the SS design itself. Surveillance was subsequently undertaken by the Curtin University team responsible for baseline surveillance (Callan et al. 2011; Majer et al. 2013).

Due to planning changes in the Gorgon project, surveillance plans for the first year were implemented partially, in two surveys called the 'Seismic Survey' and the 'Construction Survey', which were scaled down from the original in proportion to the areas of the risk zones. The first year (2010) was a drought and as rainfall in BWI is low and very seasonal, invertebrate detectability was expected to be very low, so, on the advice of surveillance biologists, the surveillance period was extended beyond a year, to include rainfall events in early 2011.

At each entry point, each species detected was identified and recorded, but multiple individuals of the one species were not counted. Species detections were compared to the baseline and if a new (non-baseline) species (NBS) was found, careful consideration was given to whether it was probably an unrecorded indigenous species or an NIS (Majer et al. 2013). It is expected that new species will be added to the baseline in diminishing numbers each year (Callan et al. 2011).

Analysis of surveillance has two parts. Of regulatory interest is whether the surveillance has been performed to requirement. This was determined by inputting the reported numbers of SSC units into the design spreadsheet and recalculating the power. Thus, if the practicalities of implementation led to changes in how many units of each SSC were performed, this could be accommodated. The other part of the analysis is the technical performance of the SS, that is, whether the estimated power of the SS to detect an NIS given its presence was accurate. For this, we assessed the proportion of the baseline species that were detected, which should equal the design power. 


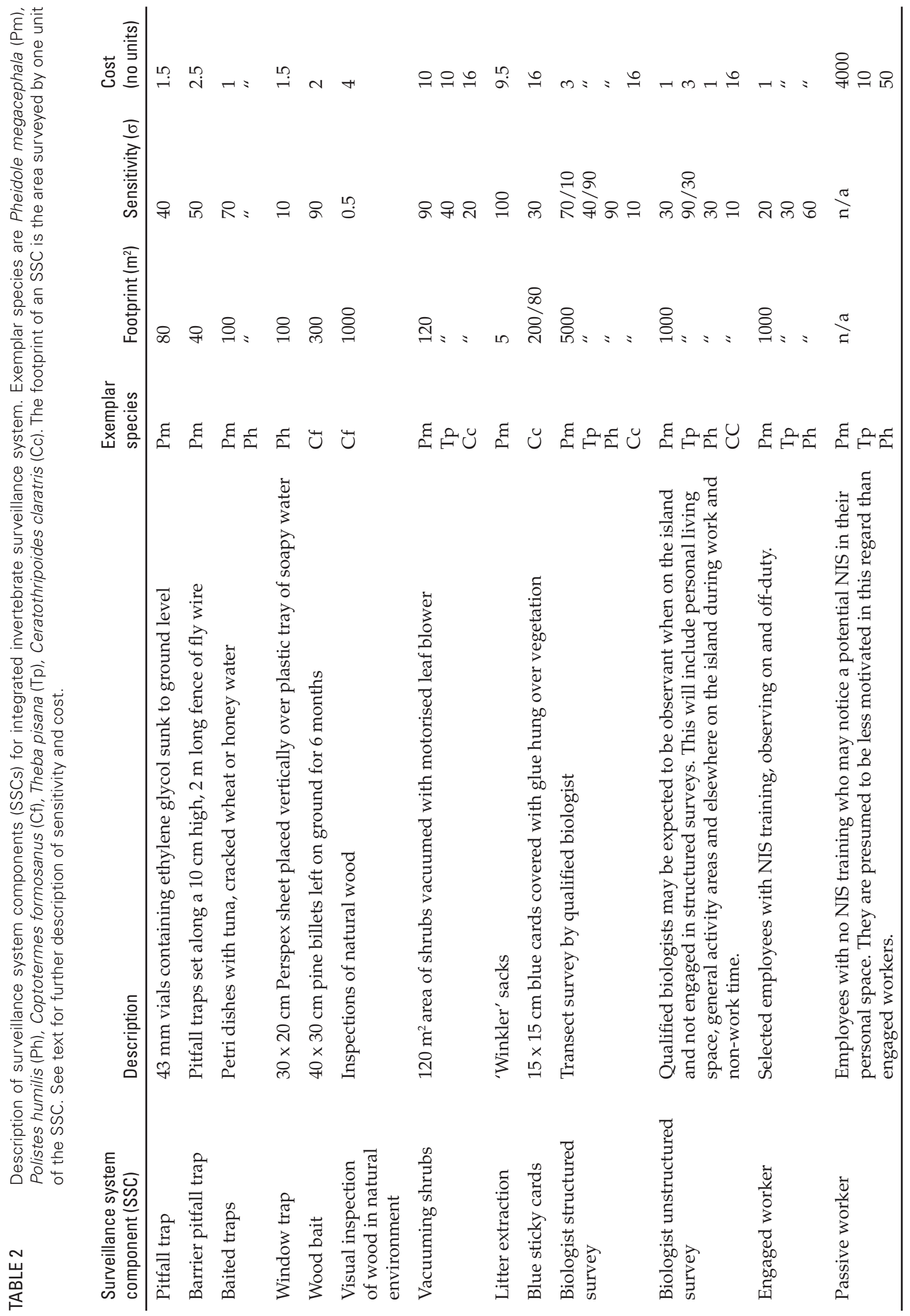




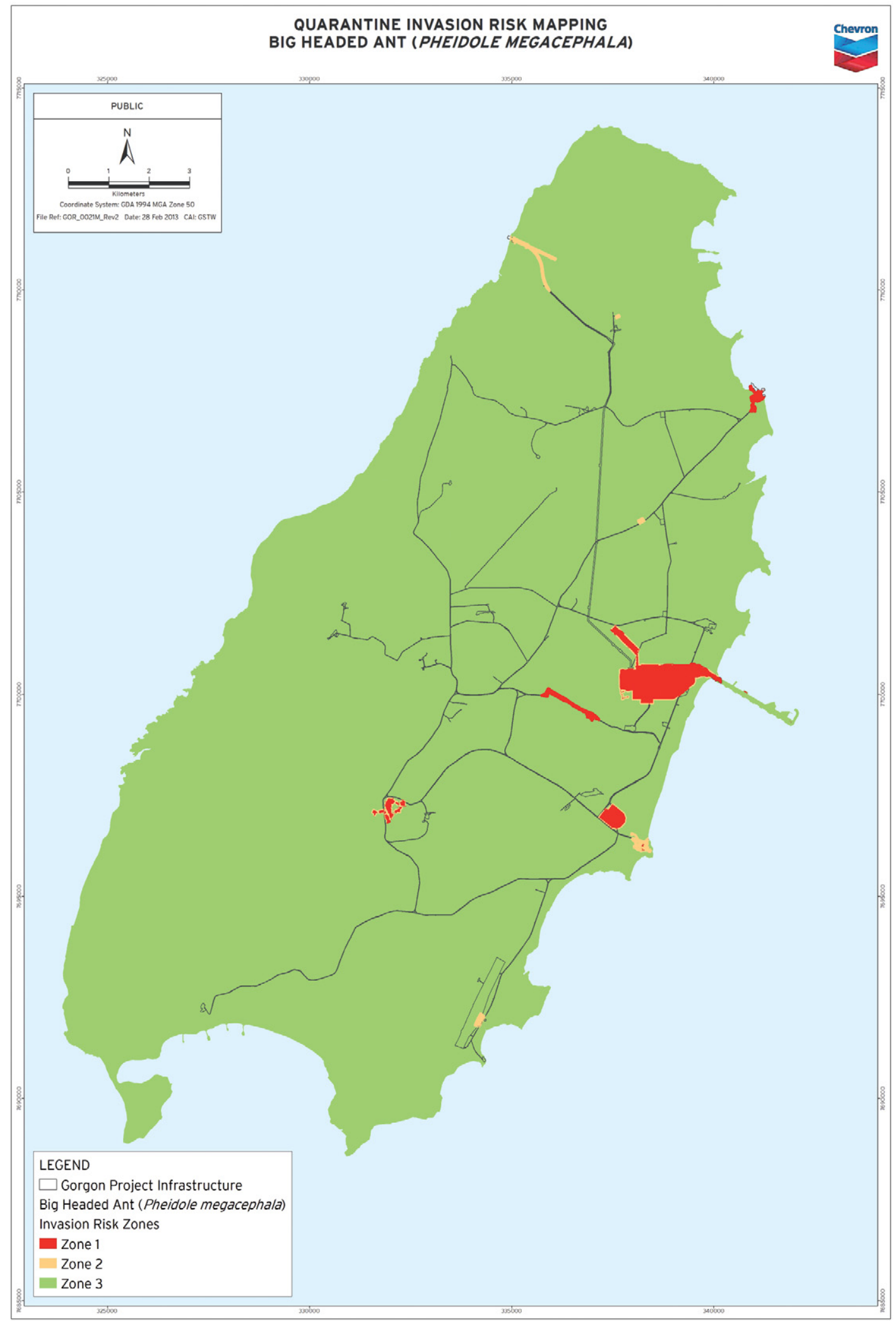

FIGURE 4

Invasion risk zoning of Barrow Island for one of five invertebrate exemplar species, the big-headed ant, Pheidole megacephala. Risk zones were determined by combining probability estimates for entry and establishment, using the Analytical Hierarchy Process. 


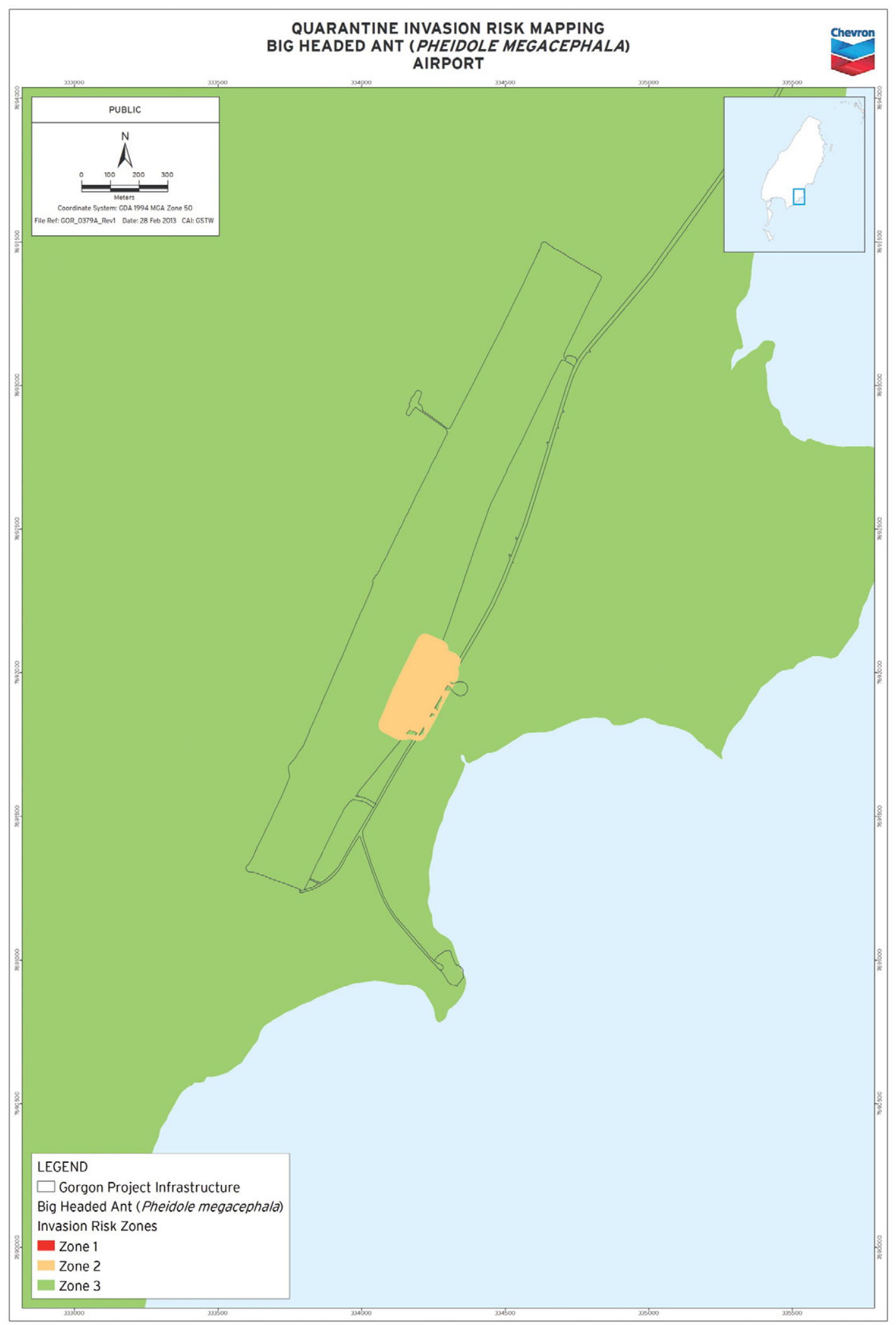

FIGURE $5 \quad$ Detail at the Airport of the invasion risk map for $P$. megacephala (see Figure 4). The runway was excluded due to its low probability of establishment. The higher risk areas are where aircraft stand and are opened, and where people congregate and move. Numbers are polygon references in the GIS system. 


\section{RESULTS}

Early in developing the SS design method, the decision tree showed us if the power requirement of 0.8 was achievable, then it supported decisionmaking on risk zones. The decision tree for $P$. megacephala (Figure 3), populated with probabilities estimated by the expert group, indicated before the SSs were devised that detection power of 0.8 was achievable. This was calculated from the probability that the NIS is not detected on entry to $\mathrm{Z1}$, stays there, establishes and is not detected:

$\operatorname{Pr}[\mathrm{D}-\mid \mathrm{P}]=0.2 \times 0.9 \times 0.8 \times 0.4=0.06$

The null hypothesis is that the NIS is absent, so $\operatorname{Pr}[\mathrm{D}-\mid \mathrm{P}]$ is accepting the null when it is false. Thus:

Power of detection $\operatorname{Pr}[\mathrm{D}+\mid \mathrm{P}]=1-0.06=0.94$

If the NIS establishes in Z2 rather than Z1, the power is near $1.0(1-0.001)$. A sensitivity analysis showed that the input parameter estimates could be varied within a reasonable range, with the overall power of the detection system remaining well above 0.8 . Power would be reduced below this level if significant changes were made to both the inspection system at the point of arrival and the detection system after entry of an NIS.

After considering the decision tree, the experts decided to allocate $90 \%$ of the SS to Z1 and $10 \%$ to $\mathrm{Z} 2$ on the basis of areas. This was more conservative than suggested by the tree, in which the probability of detection in Z2 if the NIS has left Z1 is about 90 times less than if the NIS stays in $\mathrm{Z} 1$ and is detected there. This reflected their uncertainty about the model and their concern that an undetected incursion of an NIS to Zone 2, even though very unlikely, could be a serious problem.

The five exemplar species selected were: bigheaded ant, P. megacephala; white Italian snail, Theba pisana (Müller); paper wasp, Polistes humilis (Fabricius); Formosan termite, Coptotermes formosanus Shiraki; and Oriental tomato thrips, Ceratothripoides claratris (Shumsher)). These species and the SSCs used to detect them are outlined below and summarised in Table 2. Table 3 shows the SSs for each of the exemplar species, with the initial calculation, and the final numbers of units after the integrated SS was deployed into entry points and risk zones, and experts had reviewed the SS and decided on capping and fixing certain SSCs. It also shows the chosen values of $K$. Invasion risk maps are shown by way of example for $P$. megacephala in Figure 4 (whole island) and Figure 5 (detail at one entry point). Risk maps for the integrated exemplar species, as provided to the implementation team, are shown in Figure 6 (whole island) and Figure 7 (detail at one entry point).

Pheidole megacephala, an ant that forages on the ground and on vegetation, is detected mainly using different types of ground-placed pitfall and barrier pitfall traps, as indicated by the proportion of total utility. These traps are comparatively inexpensive to run and to screen diagnostic samples, meaning that they tended to be up-weighted. Active and passive visual inspection, which are also inexpensive, are also very important; on reviewing the initial SS, the experts decided the number of units of passive worker, who may find this ant to be a domestic nuisance and will be encouraged to report them, should be increased from 1511 to 4000, in recognition of the fact that many more units than this will in fact be present on the island and they have no incremental cost. None of the other SSCs were reduced in this step, due to the roundingup effect in the spatial allocation. P. megacephala can be identified tentatively by informed nonspecialists, but specimens were to be taken to enable confirmation, or identification of the many other potential NIS of ant. This SS would also capture many other taxa of ground-dwelling invertebrates.

Theba pisana, a snail that climbs and aestivates on plants and vertical structures, is detected by active and passive inspection, in which the roles of engaged and passive workers are significant due to its easy detection and identification. The experts decided not to increase the contribution of passive workers to the SS, because it would have dominated the power contribution, even though those workers were to be present in any case. The process of active searching for T. pisana will enable detection of other potential NIS that inhabit the same niches.

Polistes humilis is detected using traps that attract or incidentally capture insects that forage on the wing. It is also detected visually in active and passive methods, since it is conspicuous and can also be intrusive due to its occasional aggression and capacity to sting people, hence the contribution to the SS of biologists and workers is substantial. The initial role of baited traps and window traps to the SS was high, however, these methods result in large quantities of specimens which would strain diagnostic resources. Hence, on review, these methods, which already had a high cost rating, were capped, resulting in a reasonable increase in the contribution of visual surveillance.

Methods to detect Coptotermes formosanus (and other termites) are limited because of its phobia of light. Hence, the SS consists entirely of SSC specific to termites and construction workers have no role.

Ceratothripoides claratris is a very small insect that as an adult is capable of flying, hence its detection requires trapping methods or observation of symptoms, and needs a specimen that can be identified by a taxonomist. The SSCs for this species will result in diagnostic specimens of many other invertebrates, hence providing monitoring for a wide range of potential NIS with similar habitat. The costs of these SSCs are high due to the technical diagnostic requirement, but they are unavoidable.

The integrated SS is shown in Table 4, deployed between areas of $\mathrm{Z} 1$ and $\mathrm{Z} 2$ associated with six 


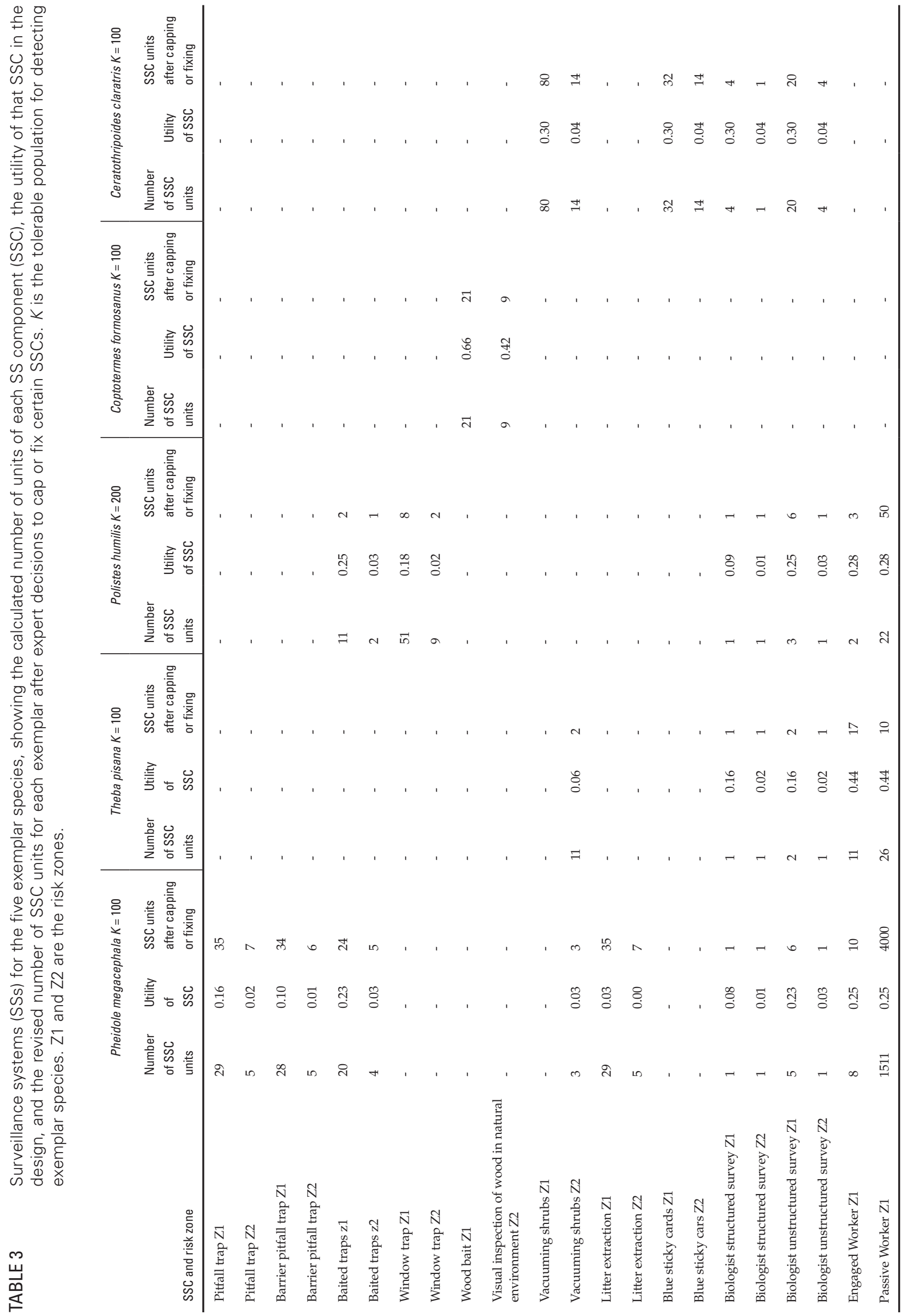




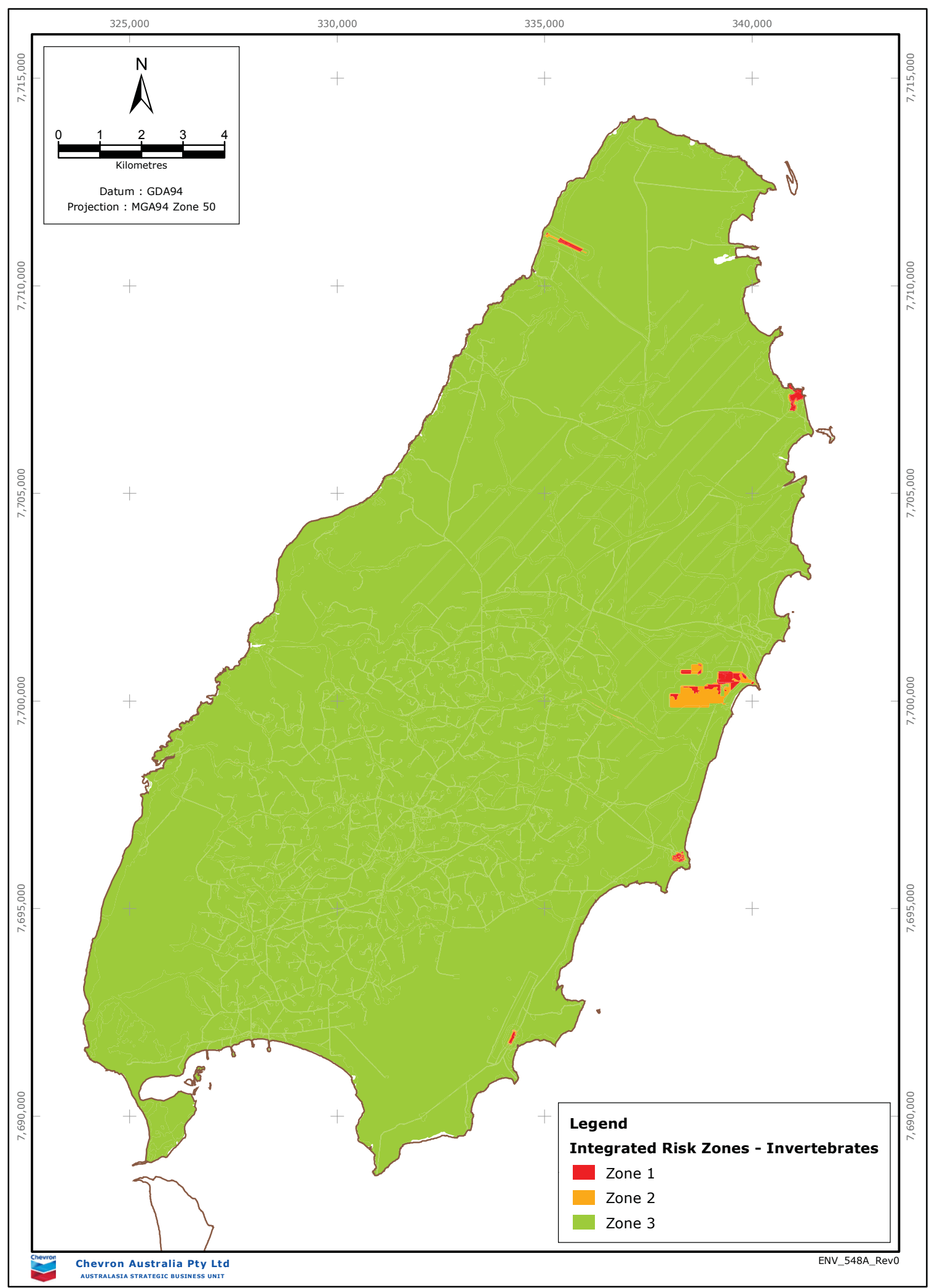

FIGURE $6 \quad$ Integrated invasion risk map of Barrow Island, for five invertebrate exemplar species. The surveillance system was deployed in Zones 1 and 2. 


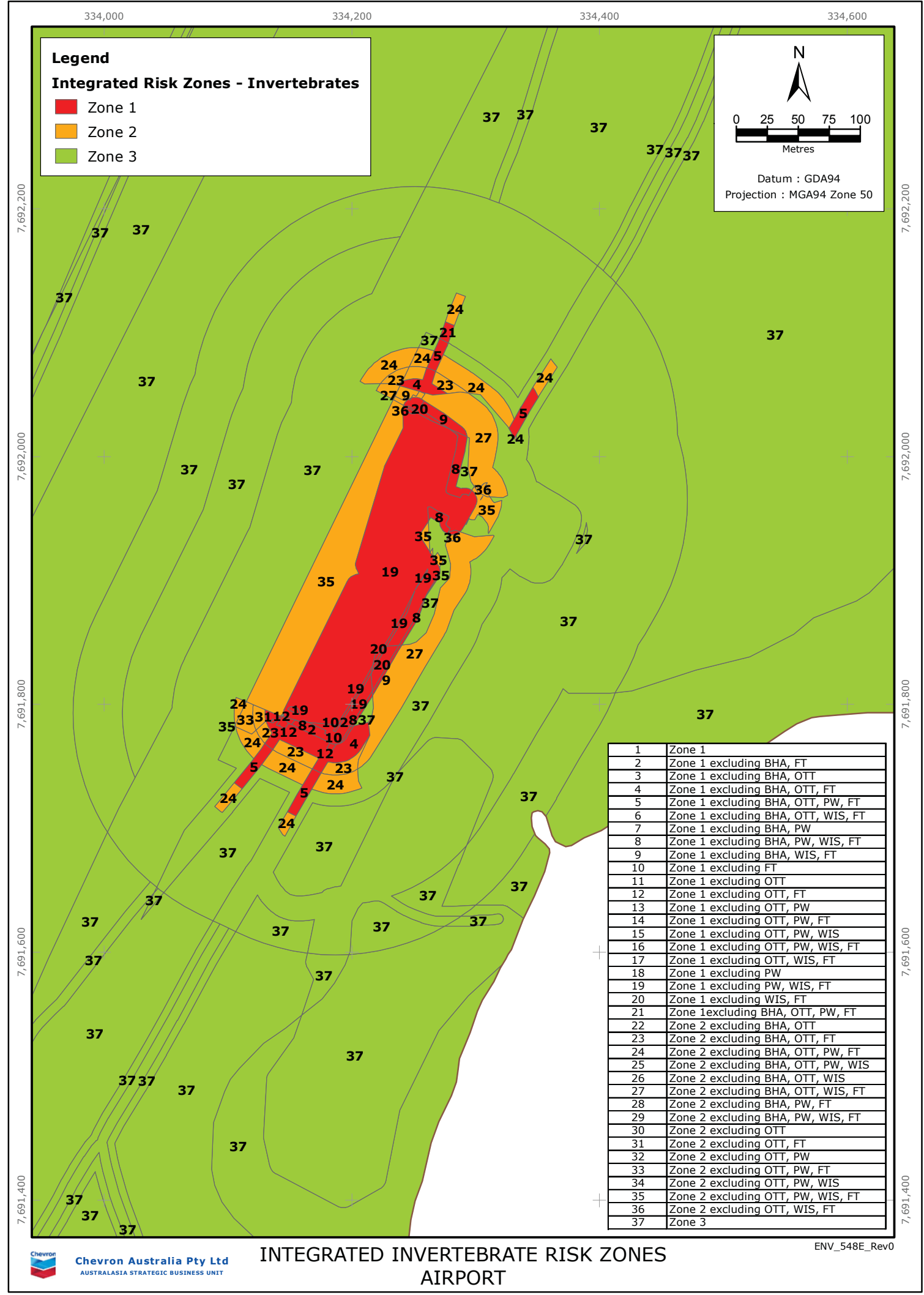

FIGURE 7

Detail of the integrated invertebrate risk map, showing the airport. Numbers refer to polygons in the GIS system. This map was provided for in-field spatial deployment of the surveillance system. 


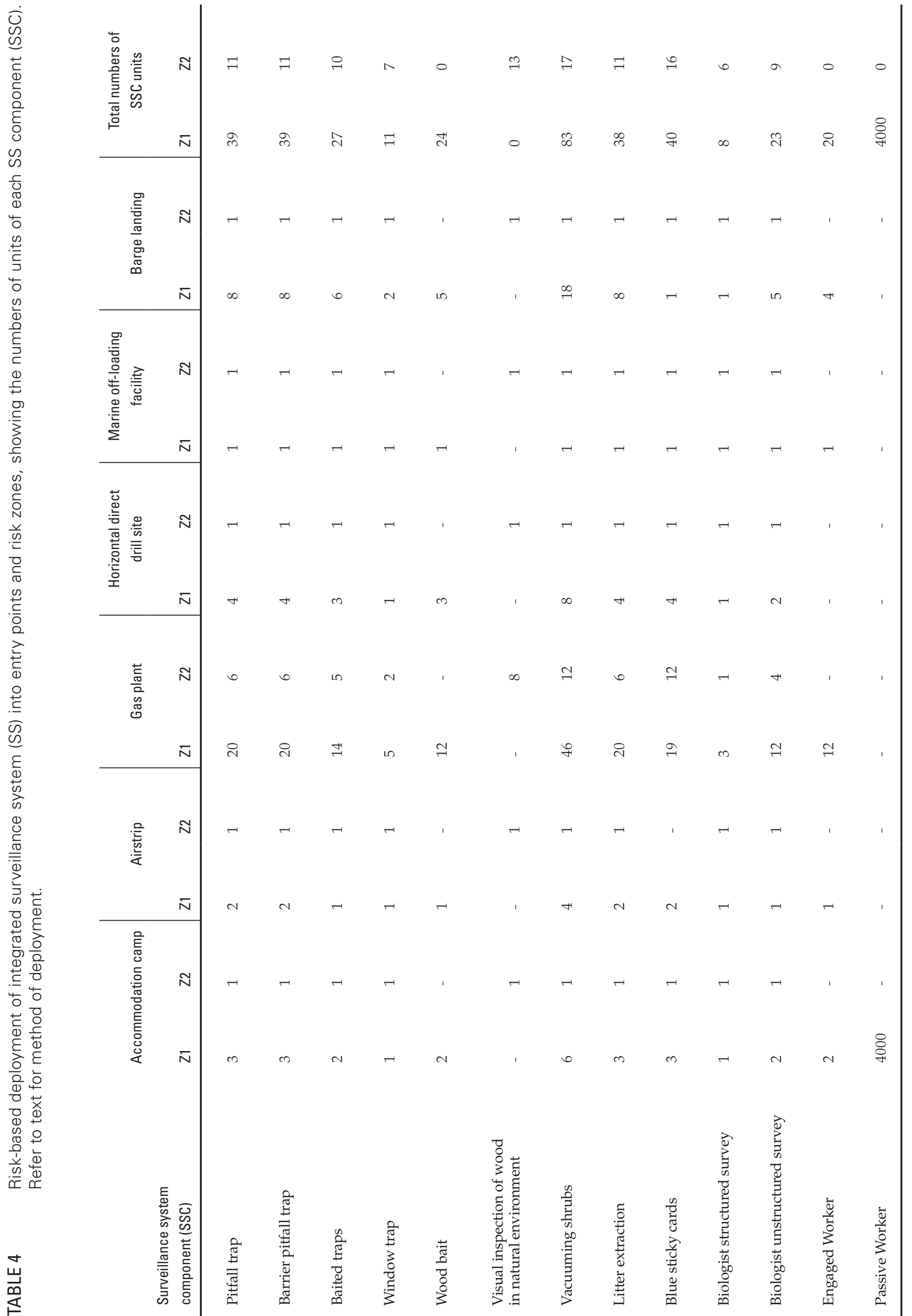


entry points (Accommodation Camp, Airstrip, Gas Plant, Horizontal Direct Drill Site, Marine Offloading Facility, Barge Landing (also known as WAPET landing)). An increase in SSC number due to rounding can be seen by comparing with Table 3 , but a direct comparison is not possible because of the overlapping of the risk zones in some cases. Although the individual SSs were all designed to a detection power of 0.8 , the integration process caused the overall surveillance frame $F$ to increase, and some Z2 areas were upgraded to Z1. Hence, the recalculated SS power for each exemplar species usually increased (post-integration powers were 1.00 for P. megacephala, T. pisana and P. humilis, 0.87 for C. formosanus and 0.92 for C. claratris). Following the first round of surveillance during 2010-11, the SS integration method was reviewed, to reduce its inflationary impact. We developed a method to allocate SSC units probabilistically, in which the proportion of the total area provided the likelihood that a unit would be allocated, giving a more equitable chance of allocating surveillance to smaller areas. After the first surveillance period, the SSCs themselves were also reviewed and changes were made, in particular substituting blue sticky cards with window traps due to heavy dust contamination, deleting shrub vacuuming and litter extraction in Zone 1 due to lack of vegetation in these heavily developed areas, and redeploying barrier pitfall traps to improve their operation and contribution to the overall SS. Lastly, the termite (C. formosanus) was removed from the integrated SS, since its SSCs were unique and its area was substantially different from the other exemplars, making it more practical to treat in a discrete SS.

The power of the surveillance actually undertaken in the truncated Seismic and Construction surveys (Table 5) exceeded the regulatory requirement, due to extra units of some SSC s being performed to compensate for those which were not performed for the reasons above. The detection power was calculated to be 0.99 for all exemplar species except C. formosanus, which was 0.92 and 0.93 in the two surveys.

Here we provide limited survey results; more detail is provided by Majer et al. (2013). No samples were determined as NIS. The Seismic Survey yielded a total of 2,450 species/entry point, representing 21 orders. Twenty-three of these findings were initially considered as NBS but on review, each was concluded to be an addition to the baseline, rather than an NIS.

The harsh seasonal conditions and the limited amount of surveillance that could be undertaken meant that the extent of re-detection of baseline species was not conclusive in regard to the robustness of surveillance. Re-detection of seven orders ranged from 10 to 61 percent.

TABLE 5

Required and actual surveillance effort (number of SSC units) for invertebrates in seismic survey and construction survey.

\begin{tabular}{lllll}
$\begin{array}{l}\text { Surveillance system } \\
\text { component (SSC) }\end{array}$ & $\begin{array}{l}\text { Seismic } \\
\text { survey design }\end{array}$ & $\begin{array}{l}\text { Seismic } \\
\text { survey actual }\end{array}$ & $\begin{array}{l}\text { Construction } \\
\text { survey design }\end{array}$ & $\begin{array}{l}\text { Construction } \\
\text { survey actual* }\end{array}$ \\
\hline Pitfall trap & 31 & 54 & 49 & 45 \\
Barrier pitfall trap & 31 & 54 & 49 & 45 \\
Baited trap & 25 & 32 & 37 & 38 \\
Wood bait & 14 & 14 & 24 & 22 \\
Visual inspection of wood (Z2) & 8 & 12 & 13 & 18 \\
Window trap & - & - & 18 & 25 \\
Vacuuming shrubs & 62 & 63 & 100 & 88 \\
Litter extraction & 33 & 40 & 49 & 41 \\
Blue sticky cards & 29 & 36 & 54 & 48 \\
Structured survey & 12 & 36 & 14 & 28 \\
Unstructured survey & 21 & 32 & 32 & 35 \\
Workerst & - & - & - & - \\
\hline
\end{tabular}

* The Construction survey actual is based on reported adjustment of the SS to the proportion of sites available. For example, if construction had not started then no surveillance was undertaken; if $20 \%$ activity, then $20 \%$ of the design SS, etc.

$\dagger$ Reports by engaged and passive workers are part of the SS, but are not active surveillance methods and hence are not reported here. 


\section{DISCUSSION}

Success (or otherwise) of the SS design method and the designs themselves that were developed for BWI can be evaluated on three criteria, whether they were:

- Accepted as satisfying the regulatory requirement for an NIS detection program with power of at least 0.8 ;

- Practical and could be implemented as planned; and

- Technically robust in terms of actual detection power equalling or exceeding the calculated power based on estimates.

The first criterion was satisfied, when the method and designs were accepted and the Gorgon project was able to proceed. Our new method enabled the power requirement to be met and simultaneously solved several complexities typical of surveillance problems, including using risk information for targeting and integrating multiple data sources. The design process benefited strongly from systematic consultation with experts, not only through bringing forth data and knowledge, and identifying areas of uncertainty and information gaps, but also enabling responsiveness to their concerns. At a number of points in the design process, the experts exhibited concern about risk, thus making some estimates that seemed conservative, for example the increased allocation of effort to Z2. The workshops allowed assumptions and concerns to be debated until consensus or some resolution was reached, so that ultimately the SS had broad support and could be recommended for approval.

The implementation of the SS during the truncated surveys in 2010-11 satisfied the second success criterion, indicating that the method and the SS design was practical and cost-effective. Developing the SS in close collaboration with experts facilitated this success, so that the design could be readily modified, to remove, add or reparameterise SSCs and their deployment at the first stage. Nevertheless, problems were experienced with some of the SSCs during implementation, but the system proved flexible and responsive, in that changes made during implementation were easily evaluated later using the spreadsheet. Cost was not a major concern during the design stage due to the tight schedule, and some steps in the process were very conservative. Several changes were made in the review to improve design efficiency and reduce inflation beyond the required power.

The third criterion of technical performance cannot be evaluated after the first year of four, as there is insufficient data to evaluate performance - a situation exacerbated by the negative effect of drought on invertebrate activity and detectability, as reflected in the low baseline re-detection rate. If the assumptions underpinning the SS design, especially $K$, footprint and $\sigma$, were overestimated or were not achieved due to inadequate performance standard, actual SS power would be reduced. Barrett et al. (2010) reported on sensitivity analysis for parameter estimates and showed that changing $\sigma$ by $10 \%$ had negligible effect on the number of units of most SSCs. Davidovitch et al. (2009) analysed robustness of the BWI SS design for P. megacephala using Info-gap decision theory (Ben-Haim 2006) and concluded that it was robust overall, but most sensitive to confidence in detection $(\sigma)$ and design prevalence $(K)$. As advised by McCann et al. (2006), when relying heavily on expert estimates, hypotheses should be formulated and tested to ensure these SSC assumptions are sound. When further data are available, statistical tools such as likelihood functions and confidence intervals (Clopper and Pearson 1934) would assist interpretation (Whittle et al. 2013).

A key factor in the cost of the SS was the choice of one year for the surveillance period. As discussed above, alternative time periods could have been considered. In the extreme, the SS could have been designed using a time period covering the full construction period of four years. In addition to being reasonable on biological grounds, the choice of an annual period provides a further element of robustness to the design, because an NIS undetected in one year may be detected in subsequent periods with the same power, or even increasing power if its population increases. The detection probability $\left(p_{t}\right)$ can be simply calculated:

$$
p_{t}=q^{t-1} p
$$

where $t$ is the time period, $p$ is the probability of detection in each period (i.e. the power) and $q$ is the probability of non-detection. Thus, if the SS detection power is 0.8 , the overall probability of detection rises to 0.96 in year 2 and to near 1.0 within five years. The one-year period was selected for this application based on consideration of the issues discussed by Martin et al. (2007), but arguably four years would have been valid. A further possible approach is to compile data from each successive year of surveillance, discounting the value of each year's information by the probability of a new incursion occurring. Principles of adaptive design could also be used, in which the design is updated based on emerging data (Sashegyi 2008).

The problem of combining alternative data sources has always dogged surveillance design. Most obviously, it is desirable to combine sources of high quality data, such as visual inspections by a biologist and specific traps. Less obviously, but a very common problem in biosecurity, is how to use surveillance data from non-specialist observations, such as those of construction workers, farmers, crop consultants and the general public. 
Cacho et al. (2010) describe the importance of this 'passive surveillance', which could also be called 'opportunistic' or 'non-authoritative', and which currently is not permissible for use in international phytosanitary surveillance for pest area freedom (IPPC 1997). Martin et al. (2007) provide the means to value this surveillance objectively and to analyse its contribution to a statement of area freedom, however, our method is the first reported way to design it into a SS.

Our SS design process for BWI enabled a systematic approach to surveillance design that could be repeated in other applications. It has now been used effectively in three domains (invertebrates, vertebrates and plants), with 17 exemplar species, each with diverse SSCs including general observations by untrained site workers. Some key steps were:

- Designing to a statistical standard (power);

- Finding a method to combine multiple data sources, which would normally tend to be ignored due to lack of such method;

- Using exemplar species to design a broadly based system with combined SSCs, rather than attempting to target pests despite high uncertainty about their identity and probability of entry;

- Developing a generalised surveillance model (Figure 2) and using this to narrow the surveillance frame by risk mapping, so that effort can be targeted affordably and effectively; and

- Overcoming information gaps using the generalised model and other tools (e.g. Figure 1, Figure 3) to develop more explicit understanding amongst the expert group.

Another useful application of our design system is to analyse existing or previous SSs designed by other methods, to calculate their power. Many of these will not have been designed to a statistical standard (Marsh and Trenham 2008), or will not have used a clear detection model (Figure 2) in order to risk-target in the surveillance frame effectively, or will not have used alternative data sources that may be available. Hence, not only may the statistical power or confidence of the SS be unknown, it may be inadequate relative to objectives. Our system can be adapted readily to analyse SSs retrospectively. We have built the prototype of a web-based tool to facilitate this, but require investment to bring this to completion.

The 'One Biosecurity' report of Beale et al. (2008) described how biosecurity risks to people, agriculture and environment are rising with increasing global interdependence. Concomitantly there is growing awareness of corporate responsibilities and the need to make decisions and allocate resources rationally (Mengersen and Whittle 2011). The Ministerial requirement for evidence-based, statistical surveillance schemes for Barrow Island such as we have described is one example of how biosecurity risks can be managed rationally and effectively, to facilitate industrial development while protecting environmental integrity.

\section{ACKNOWLEDGEMENTS}

The authors would like to acknowledge the support of the Australian Government's Cooperative Research Centres Program, which funded the project with contributions from Chevron Australia Pty Ltd. We acknowledge the contributions of experts including Peter Davis, Karl Edwards, Mike Grimm, Nihara Gunawardene, Darryl Hardie and John Scott; risk mapping support from Chevron Australia Pty Ltd (Michael Dawson and Gary Staker); and project management support from Carla Tadich.

\section{REFERENCES}

Augustin, S., De Kogel, W.J., Donner, P., Faccoli, M., Lees, D.C., Marini, L., Mori, N., Toffolo, E.P., Quilici, S., Roques, A., Yart, A. and Battisti, A. (2012). A list of methods to detect arthropod quarantine pests in Europe. EPPO Bulletin 42: 93-94.

Barrett, S., Whittle, P., Mengersen, K. and Stoklosa, R. (2010). Biosecurity threats: the design of surveillance systems, based on power and risk. Environmental and Ecological Statistics 17: 503-519.

Beale, R., Fairbrother, J., Inglis, A. and Trebeck, D. (2008). One biosecurity, a working partnership: the independent review of Australia's quarantine and biosecurity arrangements. Report to the Australian Government: Commonwealth of Australia, Canberra.

Ben-Haim, Y. (2006). Info-Gap Decision Theory: Decisions Under Severe Uncertainty, 2nd edn. Academic Press: London.

Bisevac, L. and Majer, J. (2002). Cost effectiveness and data-yield of biodiversity surveys. Journal of the Royal Society of Western Australia 85: 129-132.

Cacho, O.J., Spring, D., Hester, S. and Nally, R.M. (2010). Allocating surveillance effort in the management of invasive species: A spatially-explicit model. Environmental Modelling Software 25: 444-454.

Callan, S.K., Majer, J.D., Edwards, K. and Moro, D. (2011). Documenting the terrestrial invertebrate fauna of Barrow Island, Western Australia. Australian Journal of Entomology 50: 323-343.

Cannon, R.M. (2002). Demonstrating disease freedom - combining confidence levels. Preventive Veterinary Medicine 52: 227-249.

Cannon, R.M. and Roe, R.T. (1982). Livestock disease surveys: A field manual for veterinarians. Australian Government Publishing Service: Canberra.

Chevron Australia Pty Ltd (2011). 12: Quarantine Risks and Management. Available at: http://www. chevronaustralia.com / Libraries / Chevron Documents/gorgon_ch12_LR.pdf.sflb.ashx (accessed 
26 September 2012).

Clopper, C. and Pearson, E. (1934). The use of confidence or fiducial limits illustrated in the case of the binomial. Biometrika 26: 404-413.

Davidovitch, L., Stoklosa, R., Majer, J., Nietrzeba, A., Whittle, P., Mengersen, K. and Ben-Haim, Y. (2009). Info-Gap theory and robust design of surveillance for invasive species: The case study of Barrow Island. Journal of Environmental Management 90: 2785-2793.

De Sadeleer, N. (ed.) (2007). Implementing the precautionary principle: Approaches from the Nordic countries, EU and USA. Earthscan: London.

Denham, R. and Mengersen, K. (2007). Geographically assisted elicitation of expert opinion for regression models. Bayesian Statistics 8: 1-6.

Government of Western Australia (2007). Statement that a proposal may be implemented (pursuant to the Environmental Protection Act 1986). Gorgon Gas Development: Barrow Island Nature Reserve. Ministerial Environment Statement No. 748. State of Western Australia: Perth.

IPPC (1997). ISPM No. 6, Guidelines for Surveillance: p. 8. International Plant Protection Convention, FAO-UN: Rome.

IPPC (2007). ISPM No. 2: Framework for Pest Risk Analysis. International Plant Protection Convention, FAO-UN: Rome.

Jarrad, F., Barrett, S., Murray, J., Stoklosa, R., Whittle, P. and Mengersen, K. (2010). Ecological aspects of biosecurity surveillance design for the detection of multiple invasive animal species. Biological Invasions 13: 803-818.

Jarrad, F.C., Barrett, S., Murray, J., Parkes, J., Stoklosa, R., Mengersen, K. and Whittle, P. (2011). Improved design method for biosecurity surveillance and early detection of nonindigenous rats. New Zealand Journal of Ecology 35: 1-13.

Kolar, C.S. and Lodge, D.M. (2001). Progress in invasion biology: predicting invaders. Trends in Ecology and Evolution 16: 199-204.

Lehmann, O. and Lehmann, D. (2008). Insight Tree, Software for Decision Tree Analysis. Visionary Tools.

Low Choy, S., O'Leary, R. and Mengersen, K. (2009). Elicitation by design in ecology: using expert opinion to inform priors for Bayesian statistical models. Ecology 90: 265-277.

Majer, J.D., Callan, S. K., Edwards, K., Gunawardene, N. R. and Taylor, C. K. (2013). Baseline survey of the terrestrial invertebrate fauna. Records of the Western Australian Museum, Supplement 83: 13-112.

Marsh, D.M. and Trenham, P.C. (2008). Current trends in plant and animal population monitoring. Conservation Biology 22: 647-655.

Martin, P.A.J., Cameron, A.R. and Greiner, M. (2007). Demonstrating freedom from disease using multiple complex data sources: 1: A new methodology based on scenario trees. Preventive Veterinary Medicine 79: 71-97.

McCann, R.K., Marcot, B.G. and Ellis, R. (2006). Bayesian belief networks: applications in ecology and natural resource management. Canadian Journal of Forestry Research 36: 3053-3062.

Mehta, S.V., Haight, R.G., Homans, F.R., Polasky, S. and Venette, R.C. (2007). Optimal detection and control strategies for invasive species management. Ecological Economics 61: 237-245.

Mengersen, K. and Whittle, P. (2011). Improving accuracy and intelligibility of decisions. Journal für Verbraucherschutz und Lebensmittelsicherheit 6: 15-19.

Moore, J.L., Rout, T.M., Hauser, C.E., Moro, D., Jones, M., Wilcox, C. and Possingham, H.P. (2010). Protecting islands from pest invasion: optimal allocation of biosecurity resources between quarantine and surveillance. Biological Conservation 143: 1068-1078.

Pimentel, D., McNair, S., Janecka, J., Wightman, J., Simmonds, C., O'Connell, C., Wong, E., Russel, L., Zern, J., Aquino, T. and Tsomondo, T. (2001). Economic and environmental threats of alien plant, animal, and microbe invasions. Agriculture, Ecosystems and Environment 84: 1-20.

Quilici, S., Donner, P. and Battisti, A. (2012). Surveillance techniques for non-native insect pest detection. EPPO Bulletin 42: 95-101.

Saaty, T.L. (1987). Risk; its priority and probability: The Analytic Hierarchy Process. Risk Analysis 7: 159-172.

Salman, M.D. (2008). Surveillance and monitoring systems for animal health programs and disease surveys: pp. 3-13. In: Salman, M. (ed.), Animal disease surveillance and survey systems: Methods and applications. Iowa State Press: Ames, Iowa.

Sashegyi, A. (2008). Adaptive Design and Decision Analysis-Companions for Transforming Clinical Development. Drug Information Journal 42: 331-336.

Shmueli, G. and Fienberg, S. (2006). Current and potential statistical methods for monitoring multiple data streams for biosurveillance: pp. 109-140. In: Wilson, A., Olwell, D. and Wilson, G. (ed.). Statistical methods in counterterrorism: Game theory, modeling, syndromic surveillance, and biometric authentication. Springer: New York.

Stanaway, M., Mengersen, K. and Reeves, R. (2011). Hierarchical Bayesian modelling of early detection surveillance for plant pest invasions. Environmental and Ecological Statistics 18: 569-591.

Whittle, P., Barrett, S., Jarrad, F., Murray, J., Mengersen, K., Hardie, D., Nietrzeba, A., Stoklosa, R., Parkes, J. and Majer, J. (2009). Design of detection surveillance program for non-indigenous species of terrestrial invertebrates, plants and vertebrates on Barrow Island. Report to Chevron Australia Pty Ltd. Cooperative Research Centre for National Plant Biosecurity: Bruce, ACT.

Whittle, P.J., Jarrad, F.C., Barrett, S., Martin, P., Majer, J.D., Stoklosa, R. and Mengersen, K. (2013). A method for designing complex biosecurity surveillance systems: Detecting non-indigenous species of invertebrates on Barrow Island. Diversity and Distributions 19: 629-639.

MANUSCRIPT RECEIVED 13 DECEMBER 2012; ACCEPTED 15 APRIL 2013. 